\title{
Joint action modulates motor system involvement during action observation in 3-year-olds
}

\author{
Marlene Meyer · Sabine Hunnius · Michiel van Elk • \\ Freek van Ede $\cdot$ Harold Bekkering
}

Received: 13 December 2010 / Accepted: 24 March 2011 / Published online: 11 April 2011

(C) The Author(s) 2011. This article is published with open access at Springerlink.com

\begin{abstract}
When we are engaged in a joint action, we need to integrate our partner's actions with our own actions. Previous research has shown that in adults the involvement of one's own motor system is enhanced during observation of an action partner as compared to during observation of an individual actor. The aim of this study was to investigate whether similar motor system involvement is present at early stages of joint action development and whether it is related to joint action performance. In an EEG experiment with 3-year-old children, we assessed the children's brain activity and performance during a joint game with an adult experimenter. We used a simple button-pressing game in which the two players acted in turns. Power in the mu- and beta-frequency bands was compared when children were not actively moving but observing the experimenter's actions when (1) they were engaged in the joint action game and (2) when they were not engaged. Enhanced motor involvement during action observation as indicated by attenuated sensorimotor mu- and beta-power was found when the 3-year-olds were engaged in the joint action. This enhanced motor activation during action observation was associated with better joint action performance. The findings suggest that already in early childhood the motor system is differentially activated during action observation
\end{abstract}

M. Meyer $(\varangle) \cdot$ S. Hunnius · M. van Elk · F. van Ede .

H. Bekkering

Donders Institute for Brain, Cognition and Behaviour,

Radboud University, P.O. Box 9104,

6500 HE Nijmegen, The Netherlands

e-mail: m.meyer@donders.ru.nl

M. van Elk

Laboratory of Cognitive Neuroscience, Brain Mind Institute,

École Polytechnique Fédérale de Lausanne,

Lausanne, Switzerland depending on the involvement in a joint action. This motor system involvement might play an important role for children's joint action performance.

Keywords Joint action - Social-cognitive development . Social interaction $\cdot$ Motor system $\cdot$ Action observation

\section{Introduction}

Interacting with other human beings is a basic element of daily life, yet not a trivial challenge. Many joint actions between adults are highly sophisticated, but are performed with apparent ease (Knoblich and Jordan 2003; Sebanz et al. 2006). Cooking a meal with friends, lifting a heavy bag together, or dancing with others are only a few of numerous examples for joint actions. In the early years of life, however, children still have difficulties coordinating their actions with those of others (Brownell et al. 2006; Meyer et al. 2010). The question arises as to which mechanisms underlie the development of joint action capabilities. For adults, it has been shown that a key factor for successful joint action is the involvement of the motor system of the brain. Motor-related brain activity is not only observed during execution of one's own actions but is also important for predicting and incorporating a partner's actions (see Bekkering et al. 2009, for a review). Recent findings by Kourtis and colleagues indicate that the motor system in adults is more strongly activated when they predict actions of a joint action partner as compared to those of an individual actor. For adults, being engaged in a joint action thus has an effect on the involvement of their own motor system when observing the actions of another person (Kourtis et al. 2010). An interesting possibility to investigate the underlying neurocognitive mechanisms of action observation 
during joint action is to look at early stages of joint action in development. To date, it is unclear whether similar effects of motor system involvement can be observed when young children are engaged in a joint action. Therefore, the aim of the current study was to investigate young children's brain activity around the age that they begin to incorporate others' actions into their own action performance more successfully (cf. Meyer et al. 2010). More specifically, we examined whether being engaged in a joint action modulates the involvement of young children's own motor system when observing the actions of another person, and if so, how this motor involvement is associated with their joint action performance.

Developmental changes in joint action performance have previously been studied at a behavioral level. Early forms of joint action-such as handing over a toy-can be observed already in infancy (Carpenter 2009). However, infants' cooperation with adults often requires extensive scaffolding by the adult action partner (see e.g., Warneken and Tomasello 2007). Despite infants' motivation to act jointly with another person (see Carpenter 2009, for a review), the nature of their cooperation attempts is still not entirely mutual. It is during the second and third year of life that joint actions with peers and adults become more reciprocal and sophisticated (Brownell et al. 2006; Warneken et al. 2006). By the end of their second year of life, young children can cooperate successfully in simple interactive games like letting a ball bounce on a little trampoline by holding and moving the frame of the trampoline jointly (Warneken et al. 2006). Children aged 2 years and older reliably solve simple cooperation tasks with peers (Brownell and Carriger 1990) and show improvement in monitoring their partner's actions and integrating them into their own action performance (Brownell et al. 2006; Meyer et al. 2010). By the age of three, children have been shown to coordinate their actions in a sequential button-pressing task as accurately with an adult partner as when acting on their own (Meyer et al. 2010). At the same age, children reliably succeed in joint actions that involve complementary roles for the two action partners (Ashley and Tomasello 1998), something which appears to be difficult for younger children (Hunnius et al. 2010). In sum, the ability to successfully cooperate with others is a skill that develops gradually during early childhood.

From a neurocognitive perspective, successful joint actions require the brain to connect observed actions of others with their own motor system in order to adapt own actions accordingly. First developmental studies on action execution and action perception in young children reveal mechanisms of motor system involvement comparable to those found in adults (see e.g., Hari and Kujala 2009; Rizzolatti and Craighero 2004, for adult studies): already during infancy, motor-related brain activity has been found for action execution as well as for the observation of another person's actions (Lepage and Théoret 2006; Marshall et al. 2010; Nyström 2008; Shimada and Hiraki 2006; Southgate et al. 2010; van Elk et al. 2008).

Motor involvement during one's own actions and during the observation of others' actions can be studied by measuring oscillatory activity in the EEG signal. In both children and adults, motor activation has been associated with a power reduction in the mu- and beta-frequency bands above motor areas (Caetano et al. 2007; Hari and Kujala 2009; Marshall et al. 2010; Muthukumaraswamy and Johnson 2004; van Elk et al. 2008). A recent EEG study investigated 14-month-old infants' brain activity while they were pressing a button on their own or observing an adult doing the same (Marshall et al. 2010). During both conditions, decreased power was found in the infant equivalent of the mu-frequency range over central electrode sites. This is in line with previous findings of reduction in mu-power during action execution and observation in adults (Caetano et al. 2007; Muthukumaraswamy and Johnson 2004; Pineda et al. 2000). Moreover, the involvement of the motor system during action observation was shown to be modulated by children's action experience (van Elk et al. 2008). More motor involvement indicated by less mu- and beta-power over motor areas was found during observation of an action that children had more experience with.

Until now, developmental brain imaging studies have focused on execution and observation of individual actions. However, how young children's brain activity is modulated in the context of real-time joint action is still unexplored. Previous research in adults reveals that the involvement in a joint action has an effect on the neurocognitive processes associated with the other person's actions (see Bekkering et al. 2009, for a review). For instance, monitoring a person's action in a cooperative context showed an early component of error-related brain activation that was absent when the other person acted in a competitive context (Koban et al. 2010). In a recent set of experiments, Kourtis and colleagues (2010) investigated the involvement of the motor system when observing a joint action partner or an uninvolved actor. In one of their experiments, they created a social context in which two action partners were facing each other, while an individual actor was sitting next to them. In a Go/No-go paradigm, motor-related brain activity was assessed using EEG. A stronger decrease in beta-power and changes in motor-related potentials, which reflected stronger anticipatory motor activation, were found for one's own action partner than for the individual actor (Kourtis et al. 2010). Hence, the mere involvement in a joint activity with another person modulated the observer's motor activation when observing the partner's actions as compared to observing the actions of a person not involved in the joint action. As indicated by previous research, the involvement 
of one's own motor system plays a crucial role in understanding and predicting others' actions (see Sebanz and Knoblich 2009, for a review). When acting jointly, it is especially important to be able to understand and predict the other person's actions so that it is possible to constantly adjust one's own actions to those of the partner. Therefore, the activation of the motor system, which is thought to support action understanding and action prediction (de Lange et al. 2008; Iacoboni et al. 2005; Rizzolatti and Sinigaglia 2010; Southgate et al. 2010), is of special relevance in joint actions. Still, little is known about the role of the motor system in early joint action development.

The current study is the first to investigate motor involvement in young children who are engaged in a joint action with another person. In particular, we were interested in whether motor-related brain activity while observing another person's actions is modulated by being involved in a common joint action. Furthermore, the aim of this study was to shed light on the link between the activation of young children's motor system during action observation and their joint action performance.

In order to investigate young children's motor involvement in joint action, we simultaneously assessed 3-yearolds' brain activity and their performance in a joint action task. The experimental setup consisted of a simple computerized button-pressing game in which a cartoon figure had to be moved up a ladder by pushing two buttons alternately (cf. Meyer et al. 2010). The game was played in different conditions that varied the children's involvement in the joint action. In the joint action condition, children were playing together with an adult partner, taking turns to push the two buttons. In the joint action observation condition, the children watched the same adult play the game together with a third adult actor. To determine modulations in the child's motor system activation, we compared children's brain response during their partner's actions in the two conditions. Finally, we correlated the outcome of the EEG analysis with children's joint action performance to examine the relation between their motor system involvement for the partner's actions and their own action performance.

\section{Method \\ Participants}

The final sample consisted of seven 3 -year-old children (5 boys) with a mean age of 36.7 months $(\mathrm{SD}=.99)$. They were recruited from a database of families willing to participate in child studies. We tested another 29 children who were not included in the final sample. Seventeen of these 29 participants were excluded due to lack of or bad EEG recording traces resulting from insufficient time (i.e., lim- ited by the little participants' patience) to lower impedances in the preparation phase. Another twelve participants were excluded, either due to a lack of at least 8 movement- and artifact-free trials per condition $(n=10)$ or due to experimental errors $(n=2)$. The high dropout rate in the current experiment is consistent with other developmental studies assessing electrophysiological recordings (cf. Jeschonek et al. 2010; Leppänen et al. 2007; Southgate et al. 2010).

\section{Procedure}

During a sequential joint action game, we recorded brain activity and performance accuracy of the 3-year-old children. Figure 1 illustrates the experimental setup that consisted of a simple computerized button-pressing game proved to be suitable for children of this age in a previous study (Meyer et al. 2010). In this game, a cartoon figure of a frog could be moved up a ladder by alternately pushing two buttons. As can be seen in Fig. 1, the visual stimuli were presented on a wide-screen that was tilted to increase the height of the presented ladder and thereby the number of steps required to reach the top. In total, the ladder consisted of 42 steps that were shown on the screen. At the top of the ladder, there was a target location for the frog represented by a cartoon figure of a pig, the frog's friend on a cloud. In front of the screen, we placed two custom-made buttons to control the game and a board with the contours of two hands indicating starting and resting positions of the hands. The two buttons were interconnected via a tilt mechanism such that pushing one button down caused the other button to move up. The button presses moved the frog up the ladder. More precisely, a right button press triggered the frog to move up using its right leg and pressing the left button moved up the left leg of the frog, so that alternating left-right button presses were necessary to move the frog up. Pushing the same button more than once would not move up the frog. Button presses also elicited a short beep tone (60 ms duration) in order to keep the child's interest and attention. With each button press, EEG markers were sent such that button presses could be traced back in the EEG recordings. The computer game was implemented using Presentation (Neurobehavioral Systems, USA).

Each participant was involved in three different conditions of the game: a joint action condition, a joint action observation condition, and an individual action condition. The focus of this paper is on children's motor-related brain activity during action observation with respect to joint actions. We were interested in whether the motor system of the 3-year-olds was activated more strongly while observing others' actions when involved in a joint action as compared to watching two people acting jointly without being involved. The same person acted both as the child's joint 


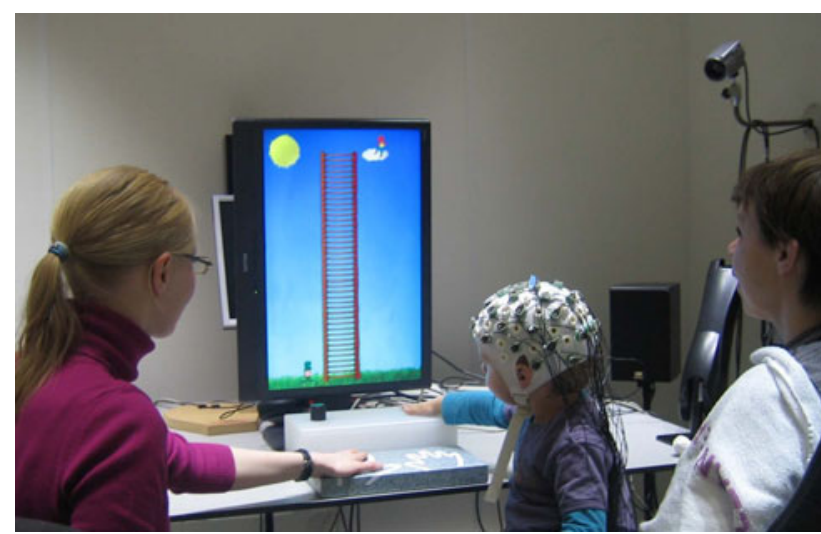

Fig. 1 The experimental setup of the joint button-pressing game. In front of a tilted wide-screen, we positioned two chess-clock buttons and resting positions marked by hand contours. By pressing the two buttons alternately, a cartoon figure could be moved up a ladder on the screen

action partner and together with a third actor in the joint action observation condition. In the current study, we concentrate on the results of the joint action and the joint action observation condition.

In the joint action condition, we instructed the children to push the right button with their right hand in turns with their adult action partner (Actor1) who pushed the left button with her left hand. More specifically, the button-pressing action was supposed to start with the hand on the resting position, which was marked by drawings of hand contours on a board in front of the buttons (see Fig. 1). Starting from this position, the action was executed by pushing the respective button and ended when the hand was placed back on the resting position. We thereby aimed to prevent children from leaving their hands on the button throughout the joint play and introduced a standardized action pattern that was comparable across conditions. During the measurement, the children sat on their parent's lap on a chair to the right of Actor1.

In the joint action observation condition, the children watched two adults (Actor1 and Actor2) playing the same game together. While Actor1 and Actor2 were playing jointly, Actor2 sat between the child and Actor1 such that the child would have to move only minimally to the right. For all children, the same first experimenter (Actor1) acted as their joint action partner. The children were not explicitly instructed where to look during the game, but in subsequent steps, only data of trials were included during which

\footnotetext{
${ }^{1}$ In the individual condition, children played the game alone pushing one of the buttons. The other button moved down automatically as it had a weight placed on it. This condition was originally meant as comparison for the joint action condition. Unfortunately, it turned out to be contaminated by a deviant movement pattern of the children elicited by the additional weight that required more force during the button press. We therefore had to omit this condition from the analysis.
}

children looked at the experimenter, the buttons, or the screen (see EEG data analysis section). Video recordings of the entire measurement session were made and aligned with the experimental events on the screen, and children's EEG and button presses were recorded.

For demonstration purposes, the joint action observation condition always preceded the joint action condition. Depending on the attention span of the children, we additionally included another run of the joint action observation condition after the joint action condition. Six out of the seven participants therefore watched Actor1 and Actor2 play both before and after they played together with Actor1. Before pooling together the data of the observation condition from the two time points, we tested for order effects. To make sure that children's motor activation did not differ significantly between the two time points, we compared activity during action observation of Actor1's button press $(t=-450$ to $0 \mathrm{~ms}$ ). Since no differences in muand beta-power were found between data collected before and after the children had played themselves (for details, see EEG data analysis), the data of the joint action observation condition were subsequently pooled.

\section{EEG recordings}

Electrophysiological recordings were conducted using childsized EEG caps with 30 electrode sites on the scalp. The Ag/ $\mathrm{AgCl}$ active electrodes were placed in an actiCap (Brain Products, Munich), arranged in the 10-20 system, and referenced to electrode $\mathrm{FCz}$ over the central midline. The signal was amplified using a 32-channel BrainAmp DC EEG amplifier, band-pass filtered $(.1-125 \mathrm{~Hz})$, and digitized at $500 \mathrm{~Hz}$. We strived to keep all impedances below $60 \mathrm{k} \Omega$.

\section{EEG data analysis}

We analyzed the data using FieldTrip, an open source Matlab (version 7.0, TheMathWorks, Inc.) toolbox developed at the Donders Institute for Brain, Cognition and Behaviour (http://www.ru.nl/neuroimaging/fieldtrip). The EEG data were locked to the button press of the first experimenter (Actor1) and determined $450 \mathrm{~ms}$ before and $450 \mathrm{~ms}$ after the button was pressed. During this time, the children were observing Actor1's actions and the effect on the screen when Actor1 was either their joint action partner (joint action condition) or the joint action partner of Actor2 (joint action observation condition). By including exclusively data from Actor1, we kept the comparison between the two conditions constant. To examine the involvement of the motor system in these two conditions, we focused on electrodes $\mathrm{C} 3$ and $\mathrm{C} 4$ over motor cortices. As mentioned in the introduction, power decrease in the $\mathrm{mu}(7-11 \mathrm{~Hz})$ - and beta(17-21 Hz)-frequency range over motor areas is associ- 
ated with motor activation (cf. Hari 2006) and thus is the focus of the current analysis. On the basis of the video recordings of the measurement session, trials were rejected if children moved their hands or did not pay attention to the game (i.e., when they looked at neither the experimenter, nor the buttons, nor the screen) during the critical period of the experimenter's action (i.e., the hand movement toward the button). Since it was an interactive game in which we relied on children's spontaneous behavior, many trials had to be excluded due to children moving during the window of interest $(t=-450$ to $0 \mathrm{~ms}$ ). Participants with less than 8 trials per condition were excluded from the analyses (see "Participants"). We visually inspected the remaining trials to exclude EEG artifacts (such as noisy channels or eye blinks). As a result, on average, 15 trials remained for the joint action condition (range 8-36) and 35 trials for the joint action observation condition (range 18-60).

A DFT filter ${ }^{2}$ was used to remove line noise from the data, and for each trial, we took out the offset by subtracting the mean signal of the entire trial. We then calculated timeresolved spectral power estimates using the Fourier transform in combination with a Hanning taper. For this, we used a $300-\mathrm{ms}$ sliding time window that was advanced in steps of $50 \mathrm{~ms}$. Power estimates were calculated for frequencies between 5 and $30 \mathrm{~Hz}$. This resulted in time-frequency representations (TFRs) of the EEG data. We obtained separate TFRs for the joint action condition and the joint action observation condition. To contrast children's brain response in these two conditions, we computed the normalized difference per time-frequency sample between the two conditions ([TFR Actor1 as joint partnerTFR Actor1 as partner of Actor2]/[TFR Actor1 as joint partner + TFR Actor1 as partner of Actor2]) (cf. van Ede et al. 2010). This normalized difference is illustrated in Fig. 2. The EEG data were locked to the button press of Actor1, which is denoted as zero. Hence, children observed Actor1 moving her hand toward the button from about $-450 \mathrm{~ms}$ to 0 . At zero, the button press of Actor 1 made the frog on the screen move upward. In the period of $0-450 \mathrm{~ms}$, children were preparing to press the button themselves in the joint action condition, while it was Actor2's turn in the joint action observation condition. At the same time, Actor1 was placing her hand back on the resting position in front of the button.

In the statistical evaluation of the electrophysiological data, we determined whether power estimates during observation of the goal-directed action of Actor 1 differed significantly between conditions. Therefore, we restricted our analyses to a priori defined time-frequency windows. We

\footnotetext{
${ }^{2}$ A DFT (discrete Fourier transform) filter takes out noise in the 50, 100 , and $150 \mathrm{~Hz}$ range, which is associated with for instance electric noise coming from light sources.
}

used the window of $450 \mathrm{~ms}$ prior to the button press until the button press $(t=-450$ to $0 \mathrm{~ms})$ and the frequency bands of $7-11 \mathrm{~Hz}(\mathrm{mu})$ and $17-21 \mathrm{~Hz}$ (beta). Values obtained from the TFR analysis (Fig. 2) were then averaged over the respective frequency range and time window. By means of one-sample $t$ tests, we examined whether these time-frequency averages were significantly different from zero. Analogous to this analysis, we evaluated the data of the joint action observation condition collected before and after the joint action condition (with $t=-450$ to $0 \mathrm{~ms}$; mu: 7-11 Hz; beta: $17-21 \mathrm{~Hz}$ ).

Due to the relatively small sample size of 7 participants, it might be argued that effects in the mu- and beta-power could be driven by extreme outliers. To exclude this possibility and to provide an overview of the strength of the observed effect in both mu- and beta-power, we ran complementary analyses on an individual participant level. For this purpose, we estimated the power in the mu- and betafrequency ranges as described earlier for each individual trial per condition and participant. To determine the average power in the two conditions for each participant, we averaged power estimates of mu-power of all trials per participant over the time window of -450 to $0 \mathrm{~ms}$. Using the same approach, we obtained average power values in the beta-frequency range for the two conditions per child. Subsequently, we evaluated the difference in power between the two conditions (separately for mu- and beta-power) on an individual participant basis using independent-sample $t$ tests with trials as units of observation.

Additionally to the general power difference on a group and individual level, we were interested in how time-locked these differences were to the actions of the joint action partner. In other words, we examined whether an increase in motor activation (reflected by less power in the mu- and beta-band) was specifically locked to Actorl's actions or rather reflected a general activation of the children's motor system. To test this, we compared the normalized difference in the mu- and beta-frequency range for the two conditions before and after Actor1 pressed the button. If the children's motor system was generally more activated in the joint action condition, we would expect no difference between these two time periods of Actor1's action. However, if the motor activation was time-locked to the actions of children's joint partner, we would expect the power differences to be different before and after Actorl's button press. Therefore, we also calculated the average normalized difference of mu-and beta-power for the time period after Actor1's button press $(t=0-450 \mathrm{~ms})$. To evaluate the statistical difference between the two time periods, we used paired-sample $t$ tests with time period (before vs. after Actor1's button press) as an independent factor, one for testing differences in the mu- and one for differences in the beta-frequency range. 
(a)
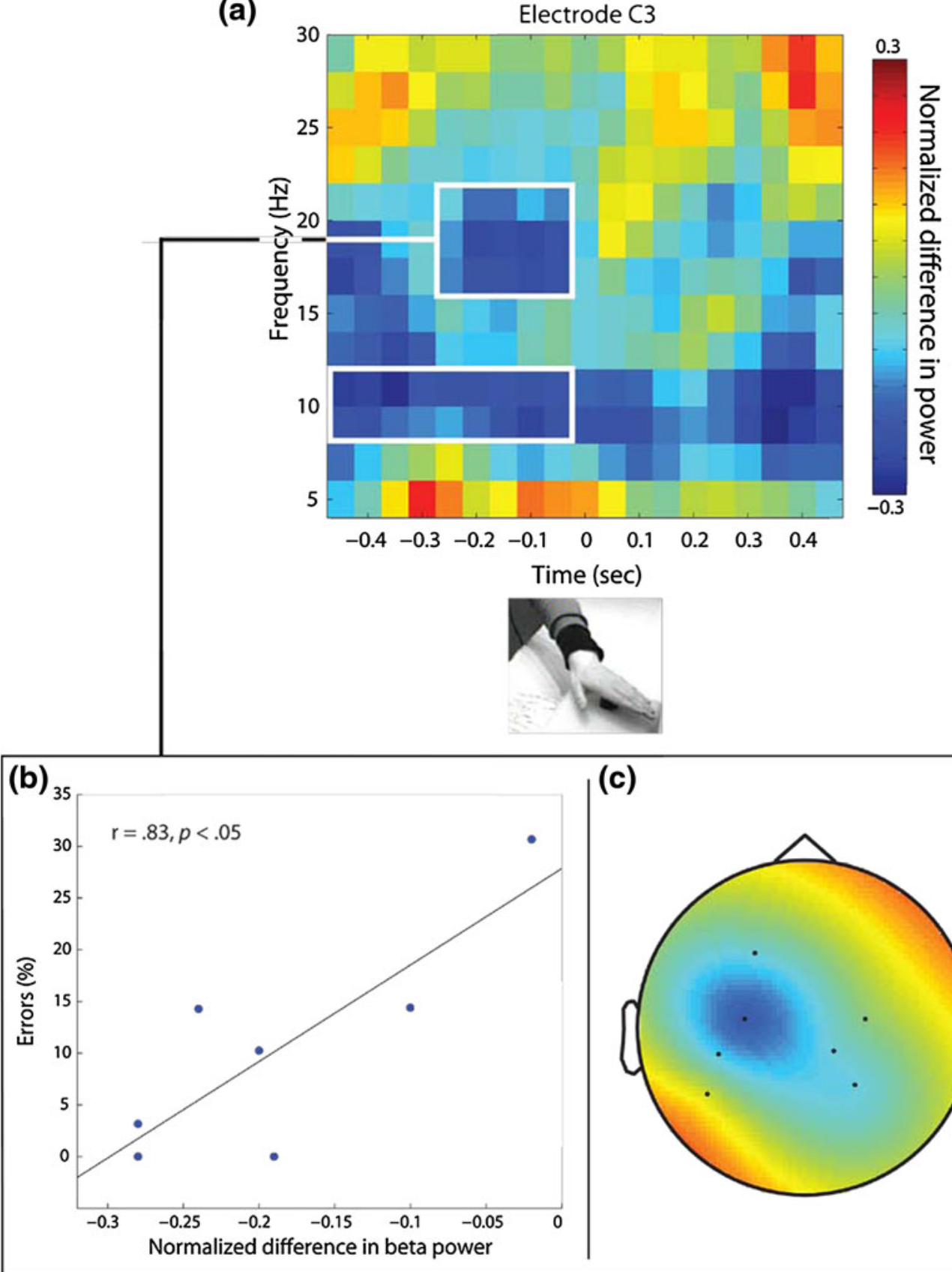

(c)

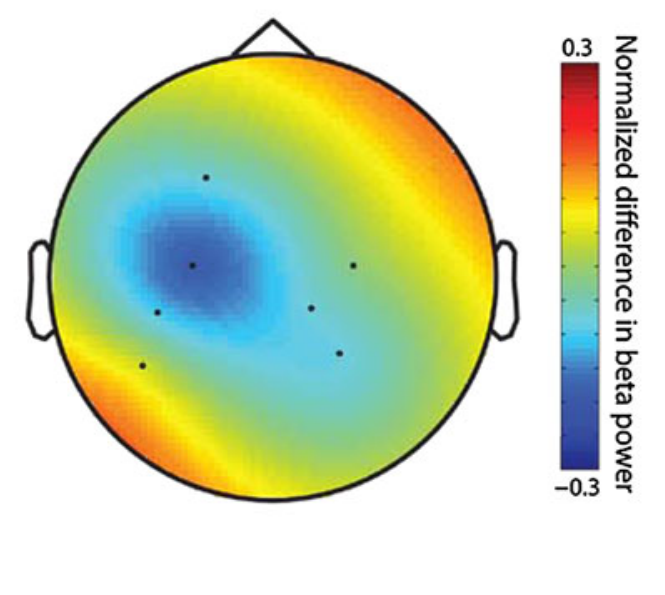

Fig. 2 a Time-resolved normalized difference in power at electrode site C3. Power differences represent the contrast between the observation of Actor 1's actions when children were involved in the joint action and when they were not involved. At time 0 , Actor 1 pushed the button that moved up a cartoon figure on the screen. Before time 0, Actor1 moved her hand toward the button. After time 0, Actor1 moved her hand back to the resting position, while it is the child's next turn in the joint action condition and Actor2's next turn in the joint action observation condition. White boxes indicate the time-frequency windows of

To further evaluate the relation of mu- and beta-power during observation of joint action with regard to children's own joint action performance, we correlated the EEG results with the behavioral button press data. On the basis the effects for which we evaluated the correlation with joint action performance and the topography (see "Methods" and "Results"). b Correlation between the individual beta-power difference and the percentage of errors children made during the joint game. Each data point represents one child. $\mathbf{c}$ Topography of the normalized beta-power difference, including the data points marked by the white box. Power differences are displayed on seven electrodes (only electrodes were used that were sufficiently noise-free for all seven children)

of the significant power differences between conditions, we chose representative time-frequency windows (see white boxes in Fig. 2). We selected the depicted time-frequency windows because they represent strongest continuous effect 
windows time-locked to the button press of Actor1. The normalized difference in power of the respective frequency and time was then averaged to obtain a representative effect value. We subsequently correlated those effect values with the percentage of errors children made when acting jointly. The percentage of errors indicates how often the 3-yearolds pushed their button when it was not their turn. The percentage was computed as number of incorrect button presses of the child (i.e., button presses when it was Actor1's turn to press) divided by the total number of times the child pushed the button when playing the joint game. We used a Pearson correlation across participants to test the relation between the effect value of each specified window (see white boxes in Fig. 2) and the percentage of errors.

\section{Results}

To examine whether young children's involvement in joint action modulates their motor activation for others' actions, we focused the EEG analysis on power differences in frequency bands (mu: 7-11 Hz, beta: $17-21 \mathrm{~Hz}$ ) and electrode sites $(\mathrm{C} 3, \mathrm{C} 4)$ associated with motor activation in the brain (cf. Pfurtscheller and Lopes da Silva 1999). We were mainly interested in the contrast between children's brain activity while observing the actions of their joint action partner (joint action condition) and of the same person acting as the joint action partner of a third person (joint action observation condition).

Figure 2 illustrates the difference in activity when children were observing Actor 1 acting as their own joint action partner and as the joint action partner of another person (Actor2). More specifically, the figure shows the normalized difference in power estimated for frequencies $5-30 \mathrm{~Hz}$ at electrode $\mathrm{C} 3$. Since results for electrode $\mathrm{C} 4$ did not show any significant difference in either of the frequency bands for the two conditions, the subsequent results only include data of electrode C3 (see topography in Fig. 2). Furthermore, there was no significant effect of order of conditions found. Data of the joint action observation condition obtained before and after the joint action condition did not differ significantly (all $P>.05$ ). Therefore, further reported results include pooled data of the joint action condition. Cold colors in the TFR of Fig. 2 represent less power for observing Actor 1 as their own joint action partner, whereas warm colors represent more power for observing Actor 1 as their joint action partner. As can be seen in Fig. 2, the difference between the conditions is most pronounced in two frequency bands, namely around $10 \mathrm{~Hz}$ and around $18 \mathrm{~Hz}$. In both bands, there is less power when children are observing their own joint action partner than when observing Actor1 playing together with a third person (Actor2). Previous studies have associated less power in these frequency ranges with more motor activation (cf. Pfurtscheller and Lopes da Silva 1999). Consequently, the current findings of decreased power indicate more motor involvement in the joint action condition compared with the joint action observation condition.

For statistical evaluation, we analyzed the power differences in the time before the button was pushed down. In both the mu- and the beta-frequency range, the normalized difference was significantly different from zero (mu, $t(6)=-3.49, \quad P=.013, \quad r=.81 ; \quad$ beta, $t(6)=-5.06$, $P=.002, r=.90)$. This indicates that the 3 -year-olds showed significantly more motor involvement when observing their own joint partner acting compared with observing the same person in joint action with another person.

Subsequently, we investigated these effects on the basis of each individual participant. Figure $3 a$ and $b$ show the resulting average power for each participant separately for the two conditions (joint action observation condition: represented in blue; joint action condition: represented in green). Individual results in the mu-frequency range are represented in Fig. 3a. The same data pattern, namely lower average power for the joint action condition compared with the joint action observation condition, can be seen in six out of seven participants. Using single-subject statistics, this tendency in mu-power differences reaches significance in two participants (Participant 1: $t(35.976)=2.39, P=.000$, $r=.37$; Participant 2: $t(55)=2.07, P=.043, r=.26$ ). Figure $3 \mathrm{~b}$ illustrates individual data for the beta-frequency range. Generally, all participants show the same data pattern as in the grand average. All seven participants exhibit the tendency of more attenuated power when observing Actor 1 in the joint action condition than in the joint action observation condition, and this difference reaches significance in three out of seven participants using single-subject statistics (Participant 2: $t(52.525)=3.29, P=.002, r=.41$; Participant 4: $t(52.592)=3.55, P=.001, r=.43$; and Participant 6: $t(24)=2.12, P=.044, r=.39)$.

Shifting the focus of the analysis back to the grand average, Fig. 2 shows that the enhanced motor activation in the mu-frequency range seems to be persistent throughout the entire time window $(900 \mathrm{~ms})$, whereas the beta-band effect appears to occur time-locked to Actor1's button press. We tested the time-specificity of the effect by comparing the time period of reaching toward the button $(t=-450$ to $0 \mathrm{~ms}$ ) with a time period of the same duration after the button had been pressed ( $t=0-450 \mathrm{~ms}$ ). Comparing the two time periods within the mu-frequency range did not show significant differences between the two time windows of observation in the grand average, $t(6)=.35, P=.737$, $r=.14$. In contrast to this, the beta-power difference was more pronounced during the goal-directed action of Actor1 than after Actor1 had pressed the button, $t(6)=-3.52$, 
Fig. 3 Power averaged over the time window of -450 to $0 \mathrm{~ms}$ in the a $\mathrm{mu}(7-11 \mathrm{~Hz})$ - and b beta(17-21 Hz)-frequency range displayed as a function of condition (joint action observation; joint action) on an individual participant level. Vertical black lines represent standard errors of the means

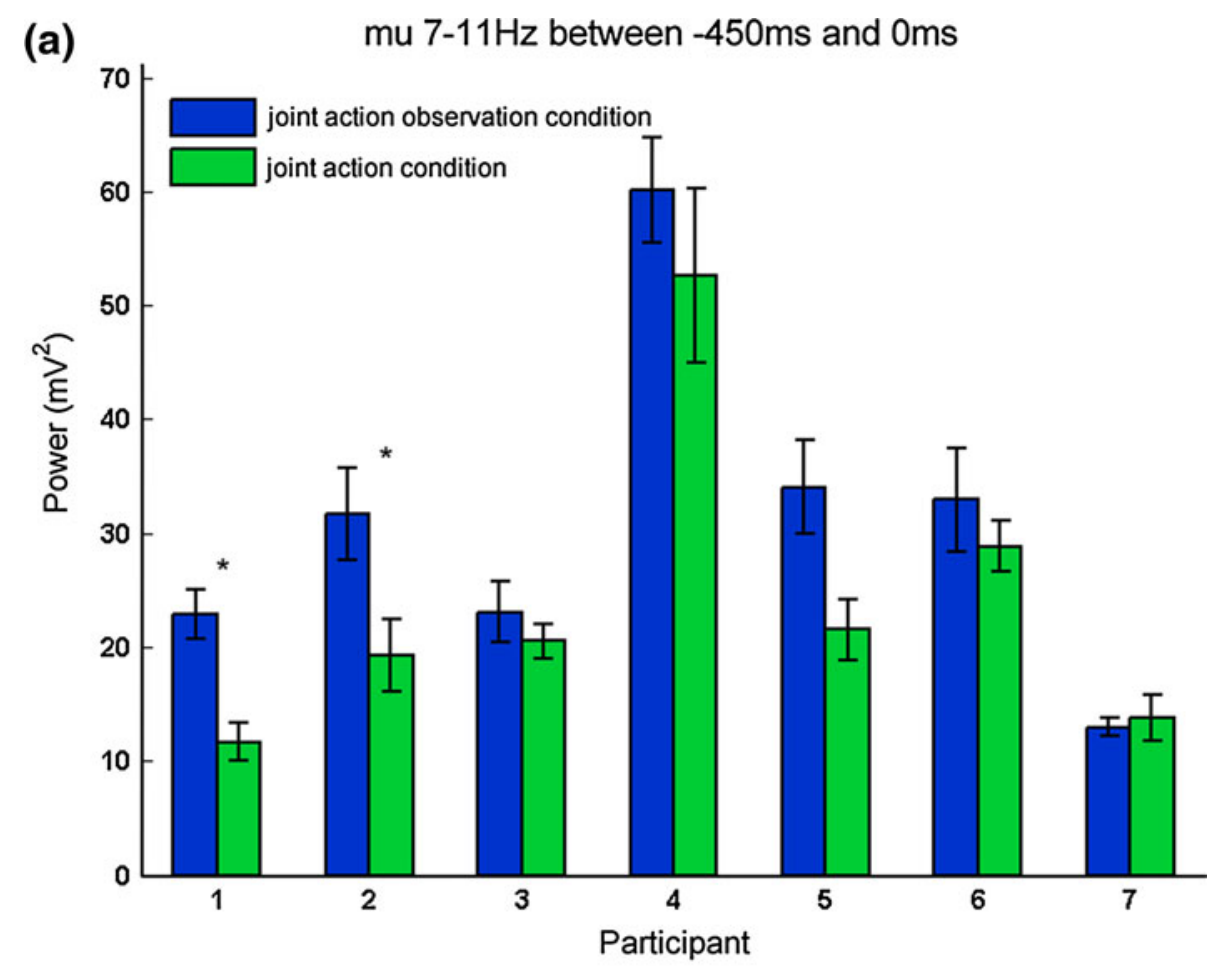

(b)

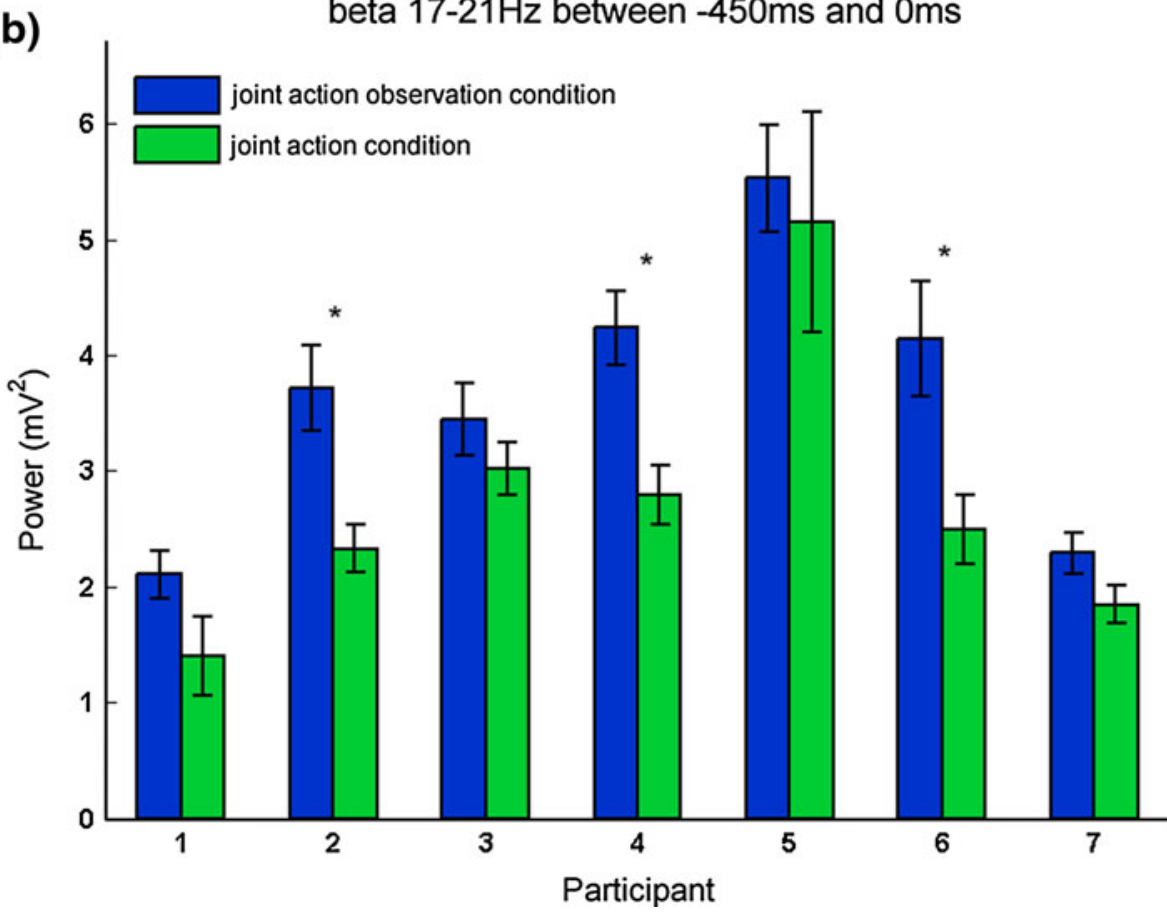

$P=.013, r=.82$. Thus, while activity in the beta-frequency range appears to be related specifically to the timing of the joint action partner's button press, the effect in the murange might be indicative of a general involvement of motor activation throughout the joint play compared with the mere observation of two people playing.

Finally, we examined the relationship between the muand beta-effects and children's joint action performance. To determine whether the enhanced motor activation in the two bands was related to how well children acted together with Actor1, we correlated the effects in the frequency bands with children's performance during joint play. For this purpose, the percentage of errors served as an indicator of performance quality. On average, the children pushed their own button about $10 \%$ of the times during the joint play (range $0-30.6 \%$ ) when it was actually the turn of 
Actor1. Based on the TFR effects (i.e., the difference between observing Actor 1 in the joint action condition and the joint action observation condition) illustrated in Fig. 2, we selected time-frequency windows that represent the strongest difference time-locked to Actor1's button press. Results revealed a significant correlation between the effect in the beta-frequency range and the percentage of errors in children's joint action performance, $r=.83, P=.021$. No significant correlation was found between the effect in the mu-frequency range and performance, $r=-.465, P>.5$.

\section{Discussion}

The present study is one of the first to explore the role of the motor system in young children's involvement in a naturalistic joint action. As hypothesized, involvement in joint action modulated activity in the motor system of 3-year-old children when observing the actions of another person. The results of the EEG analysis show significantly less power in the $\mathrm{mu}(7-11 \mathrm{~Hz})$ - and beta $(17-21 \mathrm{~Hz})$-frequency range over motor areas when children observed actions of their own joint action partner as compared to when they observed the actions of the same person playing with someone else. Since power decrease in these frequency bands is acknowledged to be associated with activation of the motor system, the current findings indicate enhanced motor system activation during action observation when the 3-year-old children were involved in the joint action. This data pattern was consistently observed over individual participants in both the mu- and beta-frequency range.

Moreover, the power decrease in the beta-frequency range appears to be time-locked to the action of children's joint action partner. After the button press of the joint action partner, power differences in this frequency band vanished. In contrast, power differences in the mu-band continued even when the partner moved her hand away from the button after having pushed it. To address possible relations between motor system involvement of the children and their joint action performance, we correlated the effects in both frequency bands with children's performance accuracy. The effect in the beta-frequency range correlated significantly with the percentage of errors children made during the joint action. This points to a negative relation between children's motor system involvement when observing their partner's actions and the amount of errors they made during the joint action: the less the child's motor system was activated during observation of their joint action partner, the more errors (i.e., erroneous button presses) the child performed. The effect in the mu-frequency range, however, turned out to be not significantly correlated to the children's joint action performance.
In general, motor activation during action observation is thought to facilitate the understanding and prediction of others' actions (Cattaneo et al. 2007; de Lange et al. 2008; Iacoboni et al. 2005; Rizzolatti and Sinigaglia 2010; Southgate et al. 2010; Stapel et al. 2010). During joint actions, it is particularly important to predict the timing and type of action to be performed by the action partner. Anticipating what our joint action partner will do next facilitates our coordination with the other person and allows for a successful interaction (see Sebanz and Knoblich 2009, for a review).

The current findings of enhanced motor activation for observing the own partner's actions as well as the correlation between motor system involvement and children's joint action performance might reflect children's predictive processing. However, it should be mentioned that the final sample size of the current experiment is rather small such that future research would be needed to further establish the observed effects. Further research is also required to make causal inferences on the function of the motor system for children's prediction of their partner's actions.

Given children's motor system involvement and its link to joint action performance, the question arises of how exactly the motor system is involved in joint actions. Does the motor activation reflect a precise spatial and temporal simulation of the other's actions? With respect to the topography, enhancement in the motor system was found in an electrode over the left hemisphere (C3) while children were observing left-hand movements. In adults, activation of left motor areas is associated with performance of right-side movements (cf. Pfurtscheller and Lopes da Silva 1999). Based on the topography of the effect, it therefore seems unlikely that the children simulated the actions of their partner on an effector level, which is in line with results of an action observation study with adults using MEG (Kilner et al. 2009). One might speculate that-while observing the action partner-the motor system of the 3-year-olds represented actions of the right hand, which was also the effector they needed for their own actions. All in all, however, the spatial resolution of the current findings is not sufficient to answer this question properly and further research is required to allow conclusive interpretations. Although the extent to which spatial aspects of the other's actions are integrated in children's motor system remains speculative, the findings show a clear integration of the action partner's timing as indicated by beta-power modulation. Integrating temporal information about the partner's actions into one's own motor system points thereby to a certain purpose enhanced motor system involvement might serve, namely facilitating action prediction in joint actions.

There may be various reasons why we found different patterns of effects for mu- compared with beta-power. One explanation for finding beta to be more time-locked 
to the partner's actions might simply be the nature of beta, which is a faster rhythm than mu. Oscillations in a higher frequency range might be more effective and flexible in adapting to events for instance by recovering faster (Pineda 2005). In fact, a recent study by van Ede and colleagues showed that modulations of beta-oscillations during somatosensory anticipation were deployed with more temporal specificity than mu-oscillations (van Ede et al. 2011). The continuous suppression of mu-power throughout the action partner's turn might reflect a general activation of the motor system bridging the time that it is not the child's turn by keeping their motor system alert. Alternative explanations might refer to differences in the function of these two rhythms as indicated by previous research. Modulations in these frequency bands might be related to the type of action or the context in which actions are performed. While beta-power during action execution and observation in adults has been shown to be modulated by the correctness of actions (Koelewijn et al. 2008), in 12-month-old infants mu-suppression has been reported to differ depending on whether the infants were observing ordinary or extraordinary actions (Stapel et al. 2010). More importantly, in adults, anticipatory suppression of the beta- but not mu-power was found to be stronger when observing actions of a partner than when observing actions of an individual actor (Kourtis et al. 2010). In accordance with this, our findings point to different modulations of mu- and beta-frequency range activity with regard to observing others' actions depending on whether one is involved in a joint action game or merely observing others playing jointly.

We have interpreted decreased power in the mu- and beta-frequency band as reflecting the activation of motorrelated areas. However, the precise neural origin of modulations in the mu- and beta-frequency bands remains a matter of debate with some evidence suggesting origins in primary motor and premotor areas, whereas others suggest more posterior (e.g., somatosensory) areas (Caetano et al. 2007; Pineda 2005; Salmelin and Hari 1994; Stancak and Pfurtscheller 1996; van Ede et al. 2010; Witham and Baker 2007). Although the precise neural sources of decrease in mu- and beta-power in the scalp-recorded EEG remain to be determined, studies in both children and adults consistently show that the execution and observation of actions is accompanied by power decreases in the mu- and beta-frequency bands (Caetano et al. 2007; Hari and Kujala 2009; Marshall et al. 2010; Muthukumaraswamy and Johnson 2004; Nyström 2008; van Elk et al. 2008). In line with these findings, the present study clearly shows that the observation of an action resulted in a decrease in mu- and beta-power, which was stronger when the 3-year-olds were engaged in joint action with the observed person as compared to when they were not.
Being engaged in a joint action appears to result in stronger motor involvement during observation of others' actions. What exactly makes a joint action situation so different from merely observing two people act together? Which factors might play a role in eliciting stronger motor involvement for others' actions when involved in a joint action? It can be speculated that motivational and attentional factors play a role here. Being involved in a joint action implies that the actions of the partner gain relevance for one's own subsequent actions. In line with this, previous research has shown that different aspects of social relevance in the relation between actor and observer, such as the identity of the actor or eye contact between actor and observer, modulate motor activation during action observation (see Frith and Frith 2010, for a review). Developmental research has shown that 3-year-olds, but not younger children, monitor their peer's actions in a selective manner (see Gauvain 2001, for a review). More precisely, 3-year-olds direct their attention to their peer's attempts to solve a task, while children below the age of three pay social attention in general to their peers without a special focus on their tasksolving activities (see Gauvain 2001, for a review). An increased attentional focus on other's task performance might thus have elicited enhanced motor involvement during the joint action. However, the extent to which attentional and motivational factors contribute to the modulation in children's motor involvement when acting jointly remains to be clarified. Moreover, in joint action, different aspects of an observed action (such as the timing) might serve as relevant cues for adapting their own action. The question arises whether in an individual action context similar nonsocial cues would subserve the same purpose and result in the same neural response. Since this question cannot be answered by the current experimental design, further investigations contrasting social and nonsocial situations are needed.

How do the current findings of 3-year-olds' brain activity relate to the development of young children's joint action performance? A recent developmental study by Gräfenhain and colleagues revealed that it is around the same age that children understand the obligations and commitments they have toward a joint action partner (Gräfenhain et al. 2009). At this age, children were also found to interact successfully when the joint action requires more complex interactions of the action partners (Ashley and Tomasello 1998). This indicates changes in children's responsiveness to the joint action partner occurring around the age of 3 years.

We have previously investigated 3-year-olds' joint action coordination in a behavioral study with a comparable joint task as used in the current experiment (Meyer et al. 2010). In this behavioral study, we found that 3-year-old children made less errors when acting with an adult action partner 
than two-and-a-half-year-olds, while both age groups performed on a similar level when playing bimanually on their own (Meyer et al. 2010). Assessing children's brain activity during a similar task and at the age when children begin to establish well-coordinated joint actions revealed that their motor system involvement during action observation was related to their joint action performance. More activation in the motor system during action observation was thereby associated with fewer errors when playing jointly. This suggests that involvement of the motor system in observing the joint action partner might play a crucial role for the development of successful joint action performance.

Together, the results show an enhanced motor activation as indicated by decreased mu- and beta-power during action observation when the 3-year-olds were involved in a joint action game with the observed actor. While power differences in the beta-range show time-locked motor activation for the partner's actions, differences in mu-power rather indicate a more general involvement of the motor system in a joint action task. Furthermore, the results show that the stronger the time-locked effect in beta-power, the fewer errors children made when acting jointly. This study is one of the first to investigate the neurocognitive mechanisms underlying joint action in young children. The present findings suggest that already in early childhood, others' actions are integrated differentially in the motor system depending on whether or not children are engaged in a joint action. This context-specific involvement of the motor system might have important consequences for developing success in joint action.

Acknowledgments We thank the children and parents for participating in this study. Moreover, we would like to thank our laboratory managers Angela Khadar and Margret van Beuningen as well as our research assistants Evelien Akker, Yvonne Maas, and Manuela Beeren. We also wish to acknowledge the technical support from Gerard van Oijen, Norbert Hermesdorf, and Pascal de Water. This research was supported by NWO-VICI grant (453-05-001) to Harold Bekkering.

Open Access This article is distributed under the terms of the Creative Commons Attribution Noncommercial License which permits any noncommercial use, distribution, and reproduction in any medium, provided the original author(s) and source are credited.

\section{References}

Ashley J, Tomasello M (1998) Cooperative problem-solving and teaching in preschoolers. Soc Dev 7(2):143-163. doi:10.1111/ 1467-9507.00059

Bekkering H, De Bruijn ERA, Cuijpers RH, Newman-Norlund R, Van Schie HT, Meulenbroek R (2009) Joint action: Neurocognitive mechanisms supporting human interaction. TopiCS 1(2):340 352. doi:10.1111/j.1756-8765.2009.01023.x

Brownell CA, Carriger MS (1990) Changes in cooperation and selfother differentiation during the second year. Child Dev 61(4):1164-1174. doi:10.1111/j.1467-8624.1990.tb02850.x

Brownell CA, Ramani GB, Zerwas S (2006) Becoming a social partner with peers: Cooperation and social understanding in one- and two-year-olds. Child Dev 77(4):803-821. doi:10.1111/j.14678624.2006.00904.x

Caetano G, Jousmaki V, Hari R (2007) Actor's and observer's primary motor cortices stabilize similarly after seen or heard motor actions. Proc Natl Acad Sci USA 104(21):9058-9062. doi:10. 1073/pnas.0702453104

Carpenter M (2009) Just how joint is joint action in infancy? TopiCS 1(2):380-392. doi:10.1111/j.1756-8765.2009.01026.x

Cattaneo L, Fabbri-Destro M, Boria S, Pieraccini C, Monti A, Cossu G, Rizzolatti G (2007) Impairment of actions chains in autism and its possible role in intention understanding. P Natl Acad Sci USA 104(45):17825-17830. doi:10.1073/pnas.0706273104

de Lange FP, Spronk M, Willems RM, Toni I, Bekkering H (2008) Complementary systems for understanding action intentions. Curr Biol 18(6):454-457. doi:10.1016/j.cub.2008.02.057

Frith U, Frith C (2010) The social brain: Allowing humans to boldly go where no other species has been. Philos $\mathrm{T} R$ Soc $\mathrm{B}$ 365(1537):165-175. doi:10.1098/rstb.2009.0160

Gauvain M (2001) The social context of cognitive development. The Guilford Press, New York

Gräfenhain M, Behne T, Carpenter M, Tomasello M (2009) Young children's understanding of joint commitments. Dev Psychol 45(5):1430-1443. doi:10.1037/a0016122

Hari R (2006) Action-perception connection and the cortical mu rhythm. In: Christa N, Wolfgang K (eds) Prog Brain Res, vol 159. Elsevier, pp 253-260

Hari R, Kujala MV (2009) Brain basis of human social interaction: From concepts to brain imaging. Physiol Rev 89(2):453-479. doi: 10.1152/physrev.00041.2007

Hunnius S, Bekkering H, Cillessen AHN (2010) The association between intention understanding and peer cooperation in toddlers. EJDS 3:368-388

Iacoboni M, Molnar-Szakacs I, Gallese V, Buccino G, Mazziotta JC, Rizzolatti G (2005) Grasping the intentions of others with one's own mirror neuron system. Plos Biol 3(3):529-535. doi:10.1371/ journal.pbio.0030079

Jeschonek S, Marinovic V, Hoehl S, Elsner B, Pauen S (2010) Do animals and furniture items elicit different brain responses in human infants? Brain Dev 32(10):863-871. doi:10.1016/j.braindev.2009.11.010

Kilner JM, Marchant JL, Frith CD (2009) Relationship between activity in human primary motor cortex during action observation and the mirror neuron system. PLoS One 4(3):e4925

Knoblich G, Jordan JS (2003) Action coordination in groups and individuals: Learning anticipatory control. J Exp Psychol Learn Mem Cogn 29(5):1006-1016. doi:10.1037/0278-7393.29.5.1006.200307955-023

Koban L, Pourtois G, Vocat R, Vuilleumier P (2010) When your errors make me lose or win: Event-related potentials to observed errors of cooperators and competitors. Soc Neurosci 4:360-374. doi:10.1080/17470911003651547

Koelewijn T, van Schie HT, Bekkering H, Oostenveld R, Jensen O (2008) Motor-cortical beta oscillations are modulated by correctness of observed action. NeuroImage 40(2):767-775. doi:10.1016/j.neuroimage.2007.12.018

Kourtis D, Sebanz N, Knoblich G (2010) Favouritism in the motor system: Social interaction modulates action simulation. Biol Lett 6(6):758-761. doi:10.1098/rsbl.2010.0478

Lepage JF, Théoret H (2006) EEG evidence for the presence of an action observation-execution matching system in children. Eur J Neurosci 23(9):2505-2510. doi:10.1111/j.1460-9568.2006. 04769.x

Leppänen JM, Moulson MC, Vogel-Farley VK, Nelson CA (2007) An ERP study of emotional face processing in the adult and infant brain. Child Dev 78(1):232-245. doi:10.1111/j.1467-8624. 2007.00994.x

Marshall PJ, Young T, Meltzoff AN (2010) Neural correlates of action observation and execution in 14-month-old infants: An 
event-related EEG desynchronization study. Dev Sci. doi:10.1111/j.1467-7687.2010.00991.x

Meyer M, Bekkering H, Paulus M, Hunnius S (2010) Joint action coordination in $2 \frac{1 / 2-}{2}$ and 3 -year-old children. Front Hum Neurosci 4:12. doi:10.3389/fnhum.2010.00220

Muthukumaraswamy SD, Johnson BW (2004) Primary motor cortex activation during action observation revealed by wavelet analysis of the EEG. Clin Neurophysiol 115(8):1760-1766. doi:10.1016/ j.clinph.2004.03.004.S1388245704001014

Nyström P (2008) The infant mirror neuron system studied with high density EEG. Soc Neurosci 3(3-4):334-347. doi:10.1080/ 17470910701563665

Pfurtscheller G, Lopes da Silva FH (1999) Event-related EEG/MEG synchronization and desynchronization: basic principles. Clin Neurophysiol 110(11):1842-1857

Pineda JA (2005) The functional significance of mu rhythms: Translating "seeing" and "hearing" into "doing". Brain Res Rev 50(1):57-68. doi:10.1016/j.brainresrev.2005.04.005

Pineda JA, Allison BZ, Vankov A (2000) The effects of self-movement, observation, and imagination on mu rhythms and readiness potentials (RP's): Toward a brain-computer interface (BCI). IEEE Trans Rehabil Eng 8(2):219-222

Rizzolatti G, Craighero L (2004) The mirror-neuron system. Annu Rev Neurosci 27:169-192. doi:10.1146/annurev.neuro.27.070203. 144230

Rizzolatti G, Sinigaglia C (2010) The functional role of the parietofrontal mirror circuit: Interpretations and misinterpretations. Nat Rev Neurosci 11(4):264-274. doi:10.1038/nrn2805

Salmelin R, Hari R (1994) Spatiotemporal characteristics of sensorimotor neuromagnetic rhythms related to thumb movement. Neuroscience 60(2):537-550

Sebanz N, Knoblich G (2009) Prediction in joint action: What, when, and where. TopiCS 1(2):353-367. doi:10.1111/j.1756-8765. 2009.01024.x

Sebanz N, Bekkering H, Knoblich G (2006) Joint action: Bodies and minds moving together. Trends Cogn Sci 10(2):70-76. doi:10.1016/j.tics.2005.12.009
Shimada S, Hiraki K (2006) Infant's brain responses to live and televised action. NeuroImage 32(2):930-939. doi:10.1016/ j.neuroimage.2006.03.044

Southgate V, Johnson MH, Karoui IE, Csibra G (2010) Motor system activation reveals infants' on-line prediction of others' goals. Psychol Sci 21(3):355-359. doi:10.1177/0956797610362058

Stancak A, Pfurtscheller G (1996) Event-related desynchronisation of central beta-rhythms during brisk and slow self-paced finger movements of dominant and nondominant hand. Brain Res Cogn Brain Res 4(3): 171-183

Stapel JC, Hunnius S, van Elk M, Bekkering H (2010) Motor activation during observation of unusual versus ordinary actions in infancy. Soc Neurosci Jul 5:1-10. doi:10.1080/17470919. 2010.490667

van Ede F, Jensen O, Maris E (2010) Tactile expectation modulates pre-stimulus [beta]-band oscillations in human sensorimotor cortex. NeuroImage 51(2):867-876. doi:10.1016/j.neuroimage.2010.02.053

van Ede F, Jensen O, Maris E (2011) Orienting attention to an upcoming tactile event involves a spatially and temporally specific modulation of sensorimotor alpha- and beta-band oscillations. J Neurosci 31(6):2016-2024. doi:10.1523/jneurosci.5630-10.2011

van Elk M, van Schie HT, Hunnius S, Vesper C, Bekkering H (2008) You'll never crawl alone: Neurophysiological evidence for experience-dependent motor resonance in infancy. NeuroImage 43(4):808-814. doi:10.1016/j.neuroimage.2008.07.057

Warneken F, Tomasello M (2007) Helping and cooperation at 14 months of age. Infancy 11(3):271-294. doi:10.1111/j.15327078.2007.tb00227.x

Warneken F, Chen F, Tomasello M (2006) Cooperative activities in young children and chimpanzees. Child Dev 77(3):640-663. doi:10.1111/j.1467-8624.2006.00895.x

Witham CL, Baker SN (2007) Network oscillations and intrinsic spiking rhythmicity do not covary in monkey sensorimotor areas. J Physiol 580(Pt.3):801-814. doi:10.1113/jphysiol.2006.124503 